\title{
Adverse systemic reaction to benznidazole
}

\author{
María Verónica Pacheco Corone ${ }^{[1]}$, Laura Oriente Frutos ${ }^{[2]}$, Eva Calabuig Muñoz ${ }^{[2]}$, \\ Diana Kury Valle ${ }^{[1]}$ and Dolores Hernández Fernández de Rojas ${ }^{[1]}$
}

[1]. Department of Allergy, Hospital Univesitari i Politècnic La Fe, Valencia, España.

[2]. Infectious Diseases Unit, Hospital Univesitari i Politècnic La Fe, Valencia, España.

\begin{abstract}
Benznidazole, drug of choice for Chagas disease (CD), has been associated with a high incidence of adverse reactions that can become serious, necessitating discontinuation of the drug. We describe the case of a Bolivian patient living in Spain for 9 years, who, following treatment with benznidazole for CD in indeterminate chronic phase, presented with fever, skin lesions, digestive symptoms, general malaise, and laboratory abnormalities. After the discontinuation of benznidazole and, the intake of antihistamines and systemic corticosteroids, the patient presented a complete resolution of the symptoms. Optimization of dose strategies and development of more effective, and better-tolerated drugs is advisable.
\end{abstract}

Keywords: Adverse drug reaction. Benznidazole. Chagas disease.

\section{INTRODUCTION}

Benznidazole is considered the first option to treat Chagas disease (CD) in Spain and is used in both the acute and chronic phases $^{1}$. The administration of the drug is associated with an elevated incidence of adverse reactions, mostly controlled with symptomatic treatment. However, some adverse reactions may become severe, necessitating discontinuation of the drug ${ }^{2}$.

Chagas disease is a parasitic disease with a high socioeconomic impact. It is endemic in Latin America, where approximately 6-8 million people are infected with Trypanosoma cruzi, and more than 100 million people live at risk of infection ${ }^{3}$. The incidence of new infections is 41,200 per annum and the mortality rate is 12,500 deaths per annum ${ }^{1}$. In recent years, CD has expanded worldwide due to migration; Spain has the highest prevalence of $\mathrm{CD}$ in Europe ${ }^{4}$.

Chagas disease usually spreads through the sting of an infected hematophagous vector (e.g., bedbugs or insects from the subfamily Triatominae). Transmission is also possible through maternal-fetal exchange, blood transfusion, and organ transplantation. These forms of transmission are more typical in non-endemic areas.

Asymptomatic individuals, with low levels of the parasite in the blood and other tissues, can still be a source of transmission ${ }^{4}$, making the diagnosis and treatment of these patients an important public health challenge ${ }^{5}$.

Corresponding author: Dra. María Verónica Pacheco Coronel.

e-mail: pacheco_marcor@gva.es

Received 1 July 2016

Accepted 22 August 2016
We describe the case of a patient who, after beginning treatment with benznidazole for $\mathrm{CD}$, presented with an adverse systemic reaction, with fever, cutaneous and digestive symptoms, as well as general malaise and laboratory abnormalities, all of which led to discontinuation of the drug.

\section{CASE REPORT}

A 49 year-old woman, originally from Bolivian (Santa Cruz), living in Spain for 9 years, serologically diagnosed in September 2014 with CD, and in the indeterminate chronic phase with no digestive or cardiac involvement, began therapy with benznidazole in April 2015. Her daily dose, which corresponded to her weight of $60 \mathrm{~kg}$, was $300 \mathrm{mg}(5 \mathrm{mg} / \mathrm{kg} / \mathrm{day})$. The patient noticed an erythematous, painful, and itchy plaque with a high edge in the left armpit 4 days after beginning treatment; this gradually increased in size. On the eighth day of treatment, she reported abdominal pain, nausea and vomiting, malaise, and dysthermia. On physical examination, she presented with low-grade fever $\left(37.6^{\circ} \mathrm{C}\right)$, erythematous papules with intense itchiness on the abdomen and lower back, a $20-\mathrm{cm}$ left axillary indurated plaque covering all of the axilla and part of the left lateral side of the chest (Figure 1), and a palpable, mobile, and painful left axillary adenopathy ( $4 \mathrm{~cm}$ in diameter). Blood analysis showed increased C-reactive protein $(70.6 \mathrm{mg} / \mathrm{L})$, elevated aspartate aminotransferase (AST) (92IU/L) and alanine aminotransferase (ALT) (39IU/L), mild neutrophilia, and eosinophilia. When her blood analysis was compared with tests previously performed by her general practitioner, eosinophilia was noted to have been present since 2011 (Figure 2). The timing of the clinical manifestations and the laboratory findings suggested the episode was drug-induced. Administration of benznidazole was suspended and the patient started receiving 


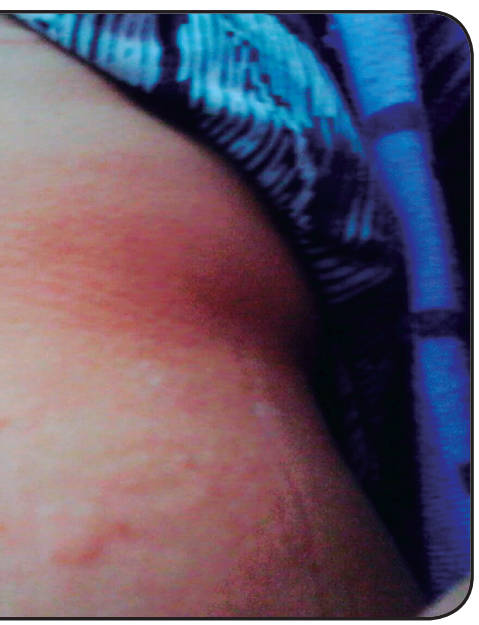

FIGURE 1 - Erythematous lesions in abdomen and indurated plaque in the left axilla and lateral side of the chest. Eight days after the beginning of treatment with benznidazole.

treatment with hydroxyzine (25mg at night-time), an extra dose of cetirizine (10mg after 12 hours if the pruritus was persistent), and prednisolone (30mg for 3 days, followed by a reduction of $10 \mathrm{mg}$ every 3 days over 9 days of treatment).

The gastrointestinal and systemic symptoms disappeared, with clear improvement of the skin lesions, 3 days after discontinuation of benznidazole. After a further 4 days, only a patch of residual post-inflammatory hyperpigmentation remained in the axilla and the adenopathy had disappeared. The patient was afebrile and laboratory parameters were within the normal range (Figure 2). After 6 months of the reaction, the patient started treatment with nifurtimox, with increasing doses for 10 days until getting the full dose $(10 \mathrm{mg} / \mathrm{kg} /$ day $)$. Seven days after starting this medication, she complained of abdominal pain and fever. Blood analysis showed leukocytosis $\left(13.24 \times 10^{3} / \mu \mathrm{l}\right)$ and increase of eosinophilia $\left(1.06 \times 10^{3} / \mu \mathrm{l}\right)$. In agreement with the patient the drug was suspended. In further revisions the patient is clinically stable, and except for the persistent eosinophilia and elevated total IgE serum levels, the laboratory parameters are normal. Stool examinations were repeatedly negative for parasites.

\section{DISCUSSION}

We report the case of an adverse reaction to benznidazole that presented with fever, malaise, skin lesions, gastrointestinal symptoms, and impaired hepatic function in a Bolivian woman in the indeterminate chronic phase of CD.

The indeterminate chronic phase of $\mathrm{CD}$ is defined as the preclinical period when the patient shows no signs or symptoms of the disease, despite positive serology. Up to $70 \%$ of patients with $\mathrm{CD}$ in the chronic phase remain in the indeterminate phase but, over time, up to $30 \%$ will develop cardiac abnormalities, between $10 \%$ and $20 \%$ digestive tract alterations (typically megaesophagus or megacolon), and 5\% neurological disorders.

Laboratory parameters

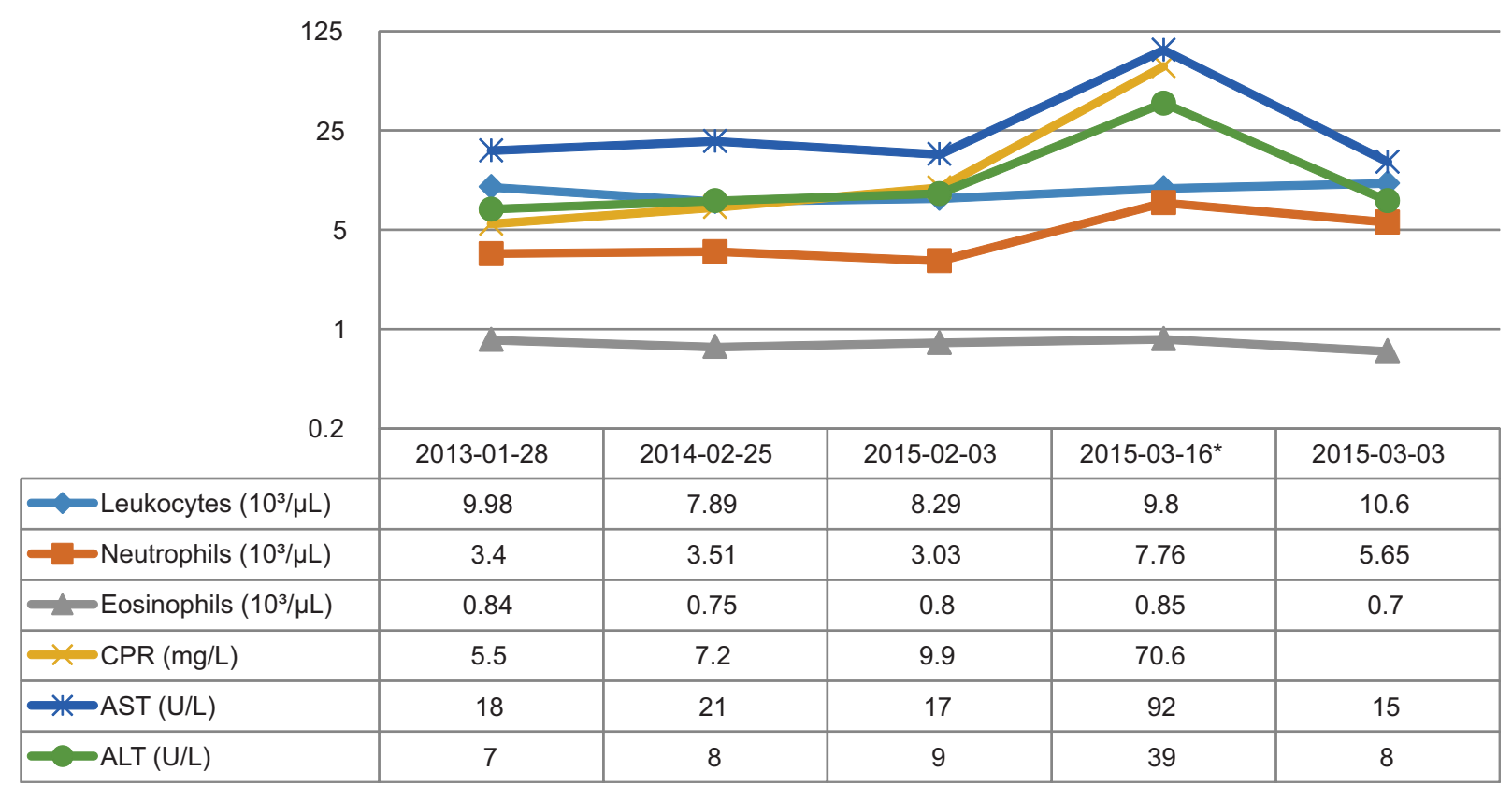

FIGURE 2 - Laboratory parameters. CRP: C-reactive protein; AST: aspartate aminotransferase; ALT: alanine aminotransferase. * Blood analysis before the discontinuation of benznidazol. 
The pharmacological treatment of $\mathrm{CD}$ has barely changed in the past 30 years. The drugs currently available are nifurtimox and benznidazole. Both drugs have variable efficacy (depending on the dose, duration of the disease, age, and origin of the patient).

Treatment in the chronic phase is controversial due to the high frequency of adverse effects ${ }^{2}$. However, benznidazole has been shown to result in negative $\mathrm{CD}$ polymerase chain reaction assays, to reduce long-term seropositivity, slow the progression of cardiomyopathy, and possibly decrease mother-to-child and blood donor-related transmission ${ }^{2,3}$.

Benznidazole, a synthetic drug of the nitroimidazole group, is the only antitrypanosomal drug currently marketed in Spain. Although in children most adverse reactions due to benznidazole are mild ${ }^{6}$, in adults the frequency of these adverse reactions necessitates discontinuation of the drug in $10-20 \%$ of cases ${ }^{7}$.

The dose for adults of $5.0-7.5 \mathrm{mg} / \mathrm{kg} /$ day for 30-60 days, divided into 2 or 3 doses per day, has been demonstrated to be effective and without toxic effects ${ }^{8}$. The primary site for benznidazole metabolism is the liver, via the cytochrome P450 system and, to a lesser extent, oxidoreductase, xanthine oxidase, and aldehyde oxidase. The mechanism of action is based on the enzymatic reduction of the nitro group, which leads to the formation of chemically active species, considered free radicals, which interact with deoxyribonucleic acid (DNA), proteins, lipids, and other cellular components ${ }^{8}$. These mechanisms are responsible for both the therapeutic action against Trypanosoma cruzi and the adverse effects.

The most common adverse reactions involve the skin and occur in up to $30 \%$ of patients; they include a variety of itchy lesions (maculopapular rash, urticaria, morbilliform exanthema, or eczema) that usually appear 10-15 days after starting benznidazole ${ }^{5,6}$. Administration of antihistamines ameliorates these symptoms. The development of exfoliative dermatitis with fever and lymphadenopathy necessitates the immediate discontinuation of benznidazole.

Even though benznidazole alone may cause painful lymph nodes, in the case we report, the rapid appearance of the initial lesion in the axilla accompanied by local adenopathy raised the possibility of other conditions, such as the skin infections cellulitis or erysipelas. However, in these conditions, the fever is usually more severe and malaise more pronounced. Furthermore, the satellite lesions and the laboratory abnormalities were not suggestive of an acute infectious process, and the lesion resolved after discontinuation the drug without the addition of antibiotics.

Due to the location of the lesion, our patient's diagnosis could have been confused with hidradenitis; however, this infection of the apocrine glands has a chronic course, including formation of abscesses. Candidiasis of the skinfolds, another possible differential diagnosis, is usually accompanied by a whitish secretion and responds only to specific treatment.

Differential diagnoses may also include severe skin reactions with systemic involvement. Drug reactions involving eosinophilia and systemic symptoms have been reported, but the eosinophilia with which our patient presented had been stable in recent years, and was possibly justified by the parasitosis itself. The cutaneous involvement did not meet the criteria for
Stevens-Johnson syndrome or toxic epidermal necrolysis. The patient did not report any trauma or insect bite in the area.

Gastrointestinal symptoms occur in $5.6 \%$ of patients, usually presenting as nausea, vomiting, epigastric pain, and decreased appetite or anorexia. Often these symptoms resolve with symptomatic treatment and are rarely a cause for discontinuation of the drug.

Benznidazole may induce elevation in the levels of transaminases and cholesterol. Our patient showed an increase in AST and ALT levels, which normalized 7 days after discontinuation of the drug (Figure 2).

Even if not in this patient, other adverse effect can occur. Peripheral neuropathy with paraesthesia often occurs at the end of treatment and is associated with toxicity ${ }^{2,8}$. Bone marrow damage caused by benznidazole is rare and necessitates immediate withdrawal of the drug. In some cases, thrombocytopenic purpura and/or leukopenia may appear ${ }^{2}$.

The case described illustrates the adverse effects, which include a variety of signs and symptoms, caused by the drug. It highlights the importance of differential diagnosis of the presenting symptoms, and the need to optimize dose strategies with the available drugs, evaluating their risk-benefit balance in the chronic phase of $\mathrm{CD}$. The development of new, more effective, and better-tolerated drugs is advisable.

\section{Conflict of interest}

The authors declare that there is no conflict of interest.

\section{REFERENCES}

1. Pérez-Molina JA, Pérez-Ayala A, Moreno S, Fernández-González MC, Zamora J, López-Velez R. Use of benznidazole to treat chronic Chagas' disease: a systematic review with a meta-analysis. J Antimicrob Chemother. 2009;64(61):1139-47.

2. Viotti R, Vigliano C, Lococo B, Alvarez MG, Petti M, Bertocchi G, et al. Side effects of benznidazole as treatment in chronic Chagas disease: fears and realities. Expert Rev Anti Infect Ther. 2009;7(2):157-63.

3. World Heath Organization (WHO). Chagas disease (American trypanosomiasis) Fact sheet, $\mathrm{N}^{\circ} 340$. WHO; 2015. Available at: http://www.who.int/mediacentre/factsheets/fs340/es/

4. Schmunis GA. Epidemiology of Chagas disease in non-endemic countries: the role of international migration. Mem Inst Oswaldo Cruz. 2007;102(Suppl I):75-85.

5. Pinazo MJ, Muñoz J, Posada E, López-Chejade P, Gállego M, Ayala E, et al. Gascon J. Tolerance of benznidazole in treatment of Chagas' disease in adults. Antimicrob Agents Chemother. 2010;54(11):4896-99.

6. Miller DA, Salvador Hernandez S, De Armas LR, Eells SJ, Traina MM, Miller LG, et al. Tolerance of benznidazole in a United States Chagas Disease Clinic. Clin Infect Dis. 2015;60(8):1237-40.

7. Urbina JA. Recent clinical trials for the etiological treatment of chronic chagas disease: advances, challenges and perspectives. J Eukaryot Microbiol. 2015;62(1):149-56.

8. Soy D, Aldasoro E, Guerrero L, Posada E, Serret N, Mejía T, et al. Population pharmacokinetics of benznidazole in adult patients with Chagas disease. Antimicrob Agents Chemother. 2015;59(6):3342-49.

9. Antinori S, Grande R, Bianco R, Traversi L, Cogliati C, Torzillo $\mathrm{D}$, et al. High frequency of adverse reactions and discontinuation with benznidazole treatment for chronic Chagas disease in Milan, Italy. Clin Infect Dis. 2015;60(12):1873-5. 